\title{
Development of Severe Fever due to Brucellosis after Cesarean: Case Report from Iran
}

\author{
Sadaf Sabzevari ${ }^{1}$, Alireza Badirzadeh ${ }^{2}$, Ateeh Kalateh ${ }^{3}$, Mohammad Seyyedin ${ }^{4}$ \\ ${ }^{1}$ Vector-borne Diseases Research Center, North Khorasan University of Medical Sciences, Bojnurd, Iran; ${ }^{2}$ Department of Medical \\ Parasitology and Mycology, School of Medicine, Shahid Beheshti University of Medical Sciences, Tehran, Iran; ${ }^{3}$ North Khorasan \\ University of Medical Sciences, Bojnurd, Iran; ${ }^{4}$ Razi Vaccine and Serum Research Institute, Mashhad, Iran.
}

\section{Corresponding Author: \\ Dr. Ateeh Kalateh \\ Email: ateeh.kalateh@yahoo.com}

This is an Open Access article distributed under the terms of the Creative Commons Attribution License (creativecommons.org/ licenses/by/3.0).

Received Accepted Published

\begin{abstract}
Background: Brucellosis, a zoonotic disease caused by Brucella spp., is an endemic infectious disease in many developing countries that causes significant economic loss. Case Report: Here, we would like to report an unusual case of human brucellosis; a 32-year-old woman who was undergone cesarean and immediately became febrile after operation. An unusual cause of post-operative fever was diagnosed brucellosis by using serologic tests. She had been treated with a combination of antimicrobials with significant clinical improvement. Conclusion: Obstetricians in endemic areas such as Iran should consider brucellosis as an unknown cause of fever.
\end{abstract}

Keywords: Brucellosis, Cesarean Section, Fever, Iran, Pregnancy, Zoonoses.

\section{Introduction}

Brucellosis, one of the major zoonotic disease, has become the world's most common bacterial disease especially in Mediterranean region and Middle Eastern countries with over 500,000 new cases annually $[1,2]$, responsible for high morbidity in infected people [3]. All parts of Iran are endemic for disease [4] and prevalence of the disease varies between $0.5 \%$ to $10.9 \%$ in different provinces [5]. Human Brucella (B) is a genus of Gram-negative Coccobacillus from the distinct genus caused by B. melitensis, B. abortus, B. canis and B. suis $[3,6]$. Brucellosis is transmitted to humans by consumption of unpasteurized dairy products or direct contact with infected animals particularly aborted fetuses and placentas [7].

It is a multi-systemic illness, with a broad clinical spectrum, ranging from asymptomatic chronic infection to severe or deadly disease [810]. Brucella infection is rare during pregnancy but may be associated with abortion, premature delivery, chorioamnionitis and intrauterine fetal death (IUFD). Incidence in pregnant women was reported about $1.3-12.2 \%$ in endemic regions $[1,11]$. Delay in diagnosis and treatment of this disease accompanied with pregnancy is common because of numerous unspecific symptoms $[9,11]$. In this paper, we report a case of pregnant woman with fever after cesarean due to brucellosis.

\section{Case Report}

We present the case of a 32-year-old pregnant woman with term breech delivery who had fever after caesarean delivery. She was referred to county hospital (Bojnurd) for elective caesarean section. Prior to the caesarean cephazolin was administered as the routine care for the disease. A healthy infant was delivered. On the first post-operative day, the patient developed a fever ranging from $38-39.5^{\circ} \mathrm{C}$ and treated by clindamycin $(600 \mathrm{mg} /$ $\mathrm{kg} / 3$ times daily) and gentamicin $(1.5 \mathrm{mg} / \mathrm{kg} /$ day $)$. On the complete examination the origin of fever was not diagnosed and she showed no response 
to the mentioned treatment. She was transferred to infectious diseases ward and examined for brucellosis but all serology results were negative. Her treatment was changed to broad-spectrum antibiotics including: clindamycin, ceftriaxone, and ampicillin. Three days post-operative, fever continued and her caesarean wound remain unhealed. Prolonged serous discharge, without purulent, was seen in the unhealed caesarean wounds incision. Wound dehiscence was obvious; therefore, fascia was sutured and skin was released without any stitches; there was no malodorous discharge. Due to opening the fascia and continuing fever uterus was suspected to be source of infection. Therefore, at the same time hysterectomy and fascia stitches were done. Recovery was satisfactory for the initial ongoing days. A few days later she returned to the same hospital with severe arthralgia, fever, chilling and sweating. The patient was hospitalized for etiological investigations. Abdominal ultrasonography, abdomino-pelvic computed tomography (CT), complete blood count (CBC), erythrocyte sedimentation rates (ESR) were in normal range but $\mathrm{C}$-reactive protein (CRP) was positive. Due to symptoms of fever and arthralgia, there was a great suspicion toward brucellosis. The serology testes for brucellosis were repeated again. All the serology tests including: serum standard tube agglutination test (Wright), Coombs Wright anti-Brucella and 2-mercaptoethanol (2ME) were positive. Wright was positive at a titer of $1 / 80$, Coombs Wright was $1 / 1280$ and $2 \mathrm{ME}$ was $1 / 640$. Treatment was done successfully with rifampicin (300 mg twice daily), doxycycline (100 mg, twice daily) and streptomycin (1g, once daily). During treatment symptoms improved, fever completely stopped, and finally wound healed.

\section{Discussion}

Human brucellosis is still one of the main challenging health problems in the Middle eastern countries like Iran that can lead to severe disabilities if it is ignored [12]. There is still controversy about the relationship between brucellosis and the outcome of pregnancy $[13,14]$. Clinical features of the disease in pregnant women are non-specific, ranging from asymptomatic to repeated classical episodes of extreme sweating, fever, and sometimes severe vaginal bleeding [1315]. Classical symptoms of brucellosis like fever in pregnant women may be mis-diagnosed and lead to disease mis-management [9]. Reports on brucellosis in pregnant woman are scarce especially with non-specific symptoms such as fever [11]. Although brucellosis could cause spontaneous abortions in human [11], fortunately, in our case a healthy infant was born.

Based on our patient's declaration she had history of sweating and joint pain without fever during pregnancy but she has not given attention. On the first post-operative day the patient was febrile and suffered from non-healing caesarean incision. This situation was constant for weeks and she failed to recover. Many tests were performed and eventually by using simple serology tests the disease was diagnosed. The principal points of current case report were that brucellosis should be considered in pregnancy and it should be in the routine evaluations for ruling out the infection. In this case, if the obstetrician and surgeon had been aware of this infection, the serum indicators of brucellosis could have tested to detect the brucella and the treatment could have been initiated prior to the hysterectomy. Therefore, increased awareness concerning brucellosis in pregnant women, its transmission, and prevention measures should be considered as a pressing need.

\section{Conclusion}

Brucellosis must be kept in mind that can cause fever in pregnancy and the disease during pregnancy should be considered a significant risk factor for adverse pregnancy outcomes in humans. Therefore, all obstetricians in endemic areas should consider brucellosis as an unknown cause of fever. We also recommend the routine simple tests of Wright, Coombs Wright and 2ME in all pregnant women who have unknown fever. 
Contributors: SS, AB: manuscript writing, literature review; AK: manuscript editing, literature review; MS: critical inputs into the manuscript. AK will act as guarantor. All authors approved the final version of this manuscript.

Funding: None; Competing interests: None stated.

\section{References}

1. Roushan MRH, Baiani M, Asnafi N, Saedi F. Outcomes of 19 pregnant women with brucellosis in Babol, northern Iran. Transactions of the Royal Society of Tropical Medicine and Hygiene. 2011;105:540-542.

2. Pappas G, Papadimitriou P, Akritidis N, Christou L, Tsianos EV. The new global map of human brucellosis. The Lancet Infectious Diseases. 2006;6:91-99.

3. Lima JIdS, Canelas CFG, Veiga ASdSB, Carvalho DMM. Neurobrucellosis and venous sinus thrombosis: an uncommon association. Revista da Sociedade Brasileira de Medicina Tropical. 2016;49:383-385.

4. Peeridogaheh H, Golmohammadi MG, Pourfarzi F. Evaluation of ELISA and Brucellacapt tests for diagnosis of human Brucellosis. Iranian Journal of Microbiology. 2013;5:14.

5. Sofian M, Aghakhani A, Velayati AA, Banifazl M, Eslamifar A, Ramezani A. Risk factors for human brucellosis in Iran: a case-control study. International Journal of Infectious Diseases. 2008;12:157-161.

6. Hasanoglu I, Guven T, Maras Y, Guner R, Tasyaran MA, Acikgoz ZC. Brucellosis as an aetiology of septic arthritis. Trop Doct. 2014;44:48-49.
7. Mahajan SK, Sharma A, Kaushik M, Raina R, Sharma S, Banyal V. Neurobrucellosis: an often forgotten cause of chronic meningitis. Trop Doct. 2016;46:54-56.

8. Dean AS, Crump L, Greter H, Schelling E, Zinsstag J. Global burden of human brucellosis: a systematic review of disease frequency. PLoS Negl Trop Dis. 2012;6:e1865.

9. Agah J, Esfehani RJ, Kamalimanesh B. Different Manifestation of Brucellosis in Pregnancy: Case Reports. Journal of Microbiology \& Infectious Diseases. 2016;6(2).

10. Rodrigues ALC, Silva SKLd, Pinto BLA, Silva JBd, Tupinambas U. Outbreak of laboratory-acquired Brucella abortus in Brazil: a case report. Revista da Sociedade Brasileira de Medicina Tropical. 2013;46:791-794.

11. Vilchez G, Espinoza M, D’Onadio G, Saona P, Gotuzzo E. Brucellosis in pregnancy: clinical aspects and obstetric outcomes. International Journal of Infectious Diseases. 2015;38:95-100.

12. Hasanoglu I, Guven T, Maras Y, Guner R, Tasyaran MA, Acikgoz ZC. Brucellosis as an aetiology of septic arthritis. Trop Doct. 2014;44:48-49.

13. Elshamy M, Ahmed AI. The effects of maternal brucellosis on pregnancy outcome. The Journal of Infection in Developing Countries. 2008;2:230-234.

14. Gulsun S, Aslan S, Satici O, Gul T. Brucellosis in pregnancy. Tropical Doctor. 2011;41:82-84.

15. Khan MY, Mah MW, Memish ZA. Brucellosis in pregnant women. Clinical Infectious Diseases. 2001;32:1172-1177. 